\title{
A CHARACTERIZATION OF THE APPROXIMATION ORDER OF TRANSLATION INVARIANT SPACES OF FUNCTIONS
}

\author{
RONG-QING JIA
}

(Communicated by J. Marshall Ash)

Abstract. Let $S$ be a space of functions on $\mathbb{R}$ with the following properties:

(i) $S$ is translation invariant, i.e., $f \in S$ implies $f(\cdot \pm 1) \in S$;

(ii) $\left.\operatorname{dim} S\right|_{[0,1]}<\infty$;

(iii) $S$ is closed under uniform convergence on compact sets.

In this paper we characterize the approximation order of $S$ by proving the following:

Theorem. $S$ provides approximation of order $k$ if and only if $S$ contains a compactly supported function $\psi$ such that the Fourier transform $\hat{\psi}$ of $\psi$ satisfies $\hat{\psi}(0)=1$ and $D^{\alpha} \hat{\psi}(2 \pi j)=0$ for $0 \leq \alpha<k$ and $j \in \mathbb{Z} \backslash\{0\}$.

This result extends a corresponding result of de Boor and DeVore, who proved the above theorem for the case $k=1$.

\section{INTRODUCTION}

Let $S$ be a space of functions on $\mathbb{R}$. Given $h>0$, let $\sigma_{h}$ be the scaling operator; i.e., for any function $f$ on $\mathbb{R}$,

$$
\sigma_{h} f:=f(\cdot / h) \text {. }
$$

We are concerned with approximation from the dilated spaces $S_{h}:=\sigma_{h} S$.

Given a positive integer $k$, we say that $S$ provides approximation of order $k$ in $L^{p} \quad(1 \leq p \leq \infty)$ if for any function $f \in W^{k, p}$, there exists a constant const (which may depend on $f$ ), such that for any $h>0$, there is $u_{h} \in S_{h}$ satisfying

$$
\operatorname{dist}\left(f, u_{h}\right) \leq \text { const } h^{k},
$$

where $\operatorname{dist}\left(f, u_{h}\right)=\left\|f-u_{h}\right\|$ in the $L^{p}$-norm. The largest integer $k$ such that the above estimate holds is said to be the approximation order of $S$. Our goal is to characterize the approximation order of $S$.

Received by the editors December 12,1988 and, in revised form, August 8, 1989.

1980 Mathematics Subject Classification (1985 Revision). Primary 41A15, 41 A25.

Key words and phrases. Order of approximation, translation invariance.

This research was partially supported by NSERC Grant No. A7687 while the author was a research associate at the University of Alberta. 
Schoenberg in his celebrated paper [5] studied what happens when $S$ is spanned by a compactly supported function $\varphi$ and its integer translates $\varphi(\cdot-j)$. To describe his result, we introduce some notation. Let $L_{\varphi}$ denote the discrete convolution operator given by

$$
L_{\varphi} f:=\sum_{j \in \mathbb{Z}} f(j) \varphi(\cdot-j) .
$$

The space of polynomials of degree $\leq k$ is denoted by $\pi_{k}$. An operator $Q$ on $\pi_{k}$ is said to be degree-reducing if for any $p \in \pi_{k}$,

$$
\operatorname{deg}(Q p)<\operatorname{deg}(p) \text {. }
$$

The identity operator is denoted by $I$. In [5], Schoenberg proved the following:

If $I-L_{\varphi}$ is degree-reducing on $\pi_{k-1}$, then $S_{\varphi}:=$ $\operatorname{span}\{\varphi(\cdot-j): j \in \mathbb{Z}\}$ provides approximation of order $k$.

However, the converse of the above statement is not true. Here is a simple example: Let $\varphi$ be 1 on $[0,1),-1$ on $[1,2)$, and zero elsewhere. Clearly, $S_{\varphi}$ provides approximation of order 1 , but $I-L_{\varphi}$ is not degree-reducing on $\pi_{0}$, the space of constants.

In the 1970s, Strang and Fix studied the space spanned by several compactly supported functions and their translates. Aware of the difficulty in characterizing the approximation order, they restricted the manner of approximation by imposing some sort of boundedness on the approximants. Such an approximation is called controlled approximation, after them. In [6], they attempted to characterize the controlled approximation order. However, their main characterization theorem was shown by Jia [4] to be incorrect. Moreover, de Boor and Jia in [3] proposed to use local approximation instead of controlled approximation and gave a complete characterization for the local approximation order.

Despite these achievements, the problem of characterization of the approximation order remains unsolved. Inspired by a recent paper of de Boor and DeVore [1], we shall solve this problem here. In [1], de Boor and DeVore considered the space $S$ of functions on $\mathbb{R}$ with the following properties:

(i) $S$ is translation invariant, i.e., $f \in S$ implies $f(\cdot \pm 1) \in S$;

(ii) $\left.\operatorname{dim} S\right|_{[0,1]}<\infty$;

(iii) $S$ is closed under uniform convergence on compact sets.

They showed that $\bigcup_{h>0} S_{h}$ is dense in $C_{0}(\mathbb{R})$ if and only if $S$ contains a good partition of unity. Their result amounts to saying that $S$ provides approximation of order 1 if and only if $S$ contains a compactly supported function $\varphi$ such that $I-L_{\varphi}$ is degree-reducing on $\pi_{0}$. We extend their result to the general case; i.e., we shall prove that $S$ provides approximation of order $k$ if and only if $S$ contains a compactly supported function $\varphi$ such that $I-L_{\varphi}$ is 
degree-reducing on $\pi_{k-1}$. This is done in $\S 3$. We first give a description of the structure of $S$, in $\S 2$.

\section{The STRUCture of THE SPACE $S$}

Let $S$ be a space of functions with the above properties (i), (ii), and (iii). Let $S^{-}$denote the subspace of $S$ consisting of those functions which vanish on $(-\infty, 0]$.

First, let us recall some results from [1].

Lemma 1. There exists a positive integer $R$ such that for any $r \in \mathbb{Z}$ and any $f \in S$ which vanishes on $[r, r+R]$ one has

$$
f \chi_{[r+R, \infty)} \in S \text { and } f \chi_{(-\infty, r]} \in S \text {. }
$$

We fix a constant $R$ which satisfies the condition of Lemma 1. Let

$$
\mu:=\left.\operatorname{dim} S\right|_{[0,1]} \text {, }
$$

and set

$$
M:=\mu R \text {. }
$$

Lemma 2. There is a finite collection $B$ of functions supported on $[0, M]$ whose restriction to $[0,1]$ forms a basis for $\left.\left(S^{-}\right)\right|_{[0,1]}$.

After de Boor and DeVore [1], we use the abbreviation

$$
b_{j}=b(\cdot-j), \quad b \in B, \quad j \in \mathbb{Z} .
$$

By length (b) we mean the smallest integer $r$ for which $\operatorname{supp} b \subseteq[i, i+r]$ for some integer $i$. We choose $B$ so that

$$
\text { length }(B):=\sum_{b \in B} \text { length }(b)
$$

is minimal.

Let $\varphi_{1}, \ldots, \varphi_{N}$ be all the elements in $B$. If $\left(a_{i}(j)\right)_{j \in \mathbb{Z}}, i=1, \ldots, N$, are real sequences, then the series

$$
\sum_{i=1}^{N} \sum_{j \in \mathbb{Z}} a_{i}(j) \varphi_{i}(\cdot-j)
$$

converges on any compact set of $\mathbb{R}$. It's sum is in $S$, since $S$ is closed under uniform convergence on compact sets. We denote by $S_{\text {loc }}$ the space of all such sums. Then $S_{\text {loc }}$ is a translation invariant subspace of $S$. It is easily seen from Lemma 2 that $S^{-}$is a subspace of $S_{\text {loc }}$. Furthermore, if $f \in S$ vanishes on $[r, r+R]$ for some integer $r$, then we know from Lemma 1 that $f \in S_{\text {loc }}$.

Lemma 3. For any interval $I_{r}:=(r, r+R)$, with $r$ an arbitrary integer and $R$ as before, the collection

$$
\Phi_{r}:=\left\{b_{j}: b \in B, \operatorname{supp} b_{j} \cap I_{r} \neq \varnothing\right\}
$$

is linearly independent over $I_{r}$. 
Suppose that $S$ is a subspace of $L^{p}(\mathbb{R}) \quad(1 \leq p \leq \infty)$. For any $f \in L^{p}(\mathbb{R})$ and a measurable subset $E$ of $\mathbb{R}$, we set

$$
\|f\|_{p, E}:=\left(\int_{E}|f|^{p}\right)^{1 / p}
$$

Often $\|f\|_{p, E}$ is abbreviated to $\|f\|_{E}$ if the exponential $p$ is clear from the context.

Set, for $r \in \mathbb{Z}$,

$$
E_{r}:=\left\{(i, j): \operatorname{supp} \varphi_{i}(\cdot-j) \cap(r, r+R) \neq \varnothing\right\} .
$$

The following lemma was also proved by de Boor and DeVore in [1].

Lemma 4. There is a positive constant const such that for any integer $r$ and any collection $\left\{c_{i, j}:(i, j) \in E_{r}\right\}$ the following inequality holds:

$$
\left|c_{i, j}\right| \leq \text { const }\left\|\sum_{k, l} c_{k, l} \varphi_{k}(\cdot-l)\right\|_{[r, r+R]} \quad, \quad \text { for all }(i, j) \in E_{r} .
$$

Let $T$ denote the translation operator given by

$$
T f=f(\cdot-1) .
$$

Using an idea from de Boor and Höllig [2], we give a description for the structure of $S$ in the following.

Theorem 1. There exists a finite dimensional subspace $V$ of $S$ such that

$$
S=V+S_{\text {loc }} \text {. }
$$

Furthermore, there exists a polynomial $q$ such that for any $f \in S$,

$$
q(T) f \in S_{\text {loc }} \text {. }
$$

Proof. Since $\operatorname{dim}\left(\left.S\right|_{[0, R]}\right)<\infty$, there exists a finite dimensional subspace $V$ of $S$ such that $\left.V\right|_{[0, R]}=\left.S\right|_{[0, R]}$. For any $f \in S$, there exists a $g \in V$ such that $f-g$ vanishes on $[0, R]$; and hence by Lemmas 1 and $2, f-g \in S_{\text {loc }}$. This proves (2.1). Suppose that $f_{1}, \ldots, f_{m}$ form a basis for $V$. For each $i$, $f_{i}, f_{i}(\cdot-1), \ldots, f_{i}(\cdot-m)$ are linearly dependent over $[0, R]$; hence there exist real numbers $a_{j}^{i}, j=0,1, \ldots, m$, not all zero, such that

$$
\sum_{j=0}^{m} a_{j}^{i} f_{i}(\cdot-j)=0 \quad \text { on }[0, R], \quad i=1, \ldots, m .
$$

Again, by Lemmas 1 and 2, we have

$$
\sum_{j=0}^{m} a_{j}^{i} f_{i}(\cdot-j) \in S_{\mathrm{loc}}, \quad i=1, \ldots, m .
$$


Let

$$
\begin{aligned}
q_{i}(t) & :=\sum_{j=0}^{m} a_{j}^{i} t^{j}, \\
q & :=\prod_{i=1}^{m} q_{i} .
\end{aligned}
$$

Then (2.3) can be restated as

$$
q_{i}(T) f_{i} \in S_{\mathrm{loc}}, \quad i=1, \ldots, m .
$$

Since $S_{\text {loc }}$ is invariant under the translation operator $T$, we have

$$
q(T) f_{i} \in S_{\mathrm{loc}}, \quad i=1, \ldots, m .
$$

Thus we conclude from (2.1) that (2.2) holds. The proof is complete.

\section{A CHARACTERIZATION OF THE APPROXIMATION ORDER}

Let $S$ be a space of functions on $\mathbb{R}$ with the above properties (i), (ii), and (iii). Suppose that $S$ is a subspace of $L^{p}(\mathbb{R}), 1 \leq p \leq \infty$. The notation $\|\cdot\|_{p, E}$ is abbreviated to $\|\cdot\|_{E}$, as it was before. For $h>0$, let $T_{h}$ be the translation operator given by

$$
T_{h} f=f(\cdot-h) .
$$

In particular, $T_{1}=T$. We define the difference operator $\nabla_{h}$ as follows:

$$
\nabla_{h}:=I-T_{h}, \quad \nabla:=\nabla_{1} .
$$

By $S^{h}$ and $S_{\text {loc }}^{h}$ we denote $\sigma_{h}(S)$ and $\sigma_{h}\left(S_{\text {loc }}\right)$, respectively. For $f \in L^{1}(\mathbb{R})$, $\hat{f}$ denotes the Fourier transform of $f$ :

$$
\hat{f}(\xi):=\int_{\mathbb{R}} e^{-i x \xi} f(x) d x .
$$

Let $B=\left\{\varphi_{1}, \ldots, \varphi_{N}\right\}$ be the collection of compactly supported functions in $S$ given in $\S 2$. Thus $S_{\text {loc }}$ is spanned by $\varphi_{i}(\cdot-j), i=1, \ldots, N, j \in \mathbb{Z}$, and Lemma 4 holds for $\left\{\varphi_{1}, \ldots, \varphi_{N}\right\}$. In this section, we characterize the approximation order of $S$. This characterization is based on the characterization given by de Boor and Jia in [3] for the local approximation order provided by $B=\left\{\varphi_{1}, \ldots, \varphi_{N}\right\}$. The following lemma can be derived from [3]:

Lemma 5. Let $k$ be a positive integer and $\left(h_{n}\right)_{n=1,2, \ldots}$ be a sequence of positive numbers which converges to 0 . For each $n=1,2, \ldots$, let $u_{n}$ be a (finite) linear combination of $\varphi_{l}\left(\dot{\dot{h_{n}}}-j\right), l=1, \ldots, N, j \in \mathbb{Z}$. Suppose that the sequence $\left(u_{n}\right)$ satisfies the following conditions:

(1) $\lim _{n \rightarrow \infty} \hat{u}_{n}(0)=1$,

(2) $\lim _{n \rightarrow \infty}\left(D^{\alpha} \hat{u}_{n}\left(\xi / h_{n}\right) / h_{n}^{k-1}\right)=0$ for $\xi \in \mathbb{R} \backslash\{0\}$ and $0 \leq \alpha<k$. 
Then there exists a function $\psi$ which is a linear combination of $\varphi_{l}, l=$ $1, \ldots, N$; and their integer translates such that $\hat{\psi}(0)=1$ and

$$
D^{\alpha} \hat{\psi}(2 \pi j)=0 \text { for } 0 \leq \alpha<k \text { and } j \in \mathbb{Z} \backslash\{0\} .
$$

Let us point out how this lemma can be derived from [3]. Since each $u_{n}$ is a (finite) linear combination of $\varphi_{l}\left(\dot{\bar{h}_{n}}-j\right)$, there exist sequences $\left(c_{l}^{n}(j)\right)_{j \in \mathbb{Z}}$, $l=1, \ldots, N$, such that

$$
u_{n}=\sum_{l=1}^{N} \sum_{j \in \mathbb{Z}} c_{l}^{n}(j) \varphi_{l}\left(\frac{\dot{h_{n}}}{h^{\prime}}-j\right)
$$

with $c_{l}^{n}(j)=0$ except for finitely many $j$. The Fourier transform of $u_{n}$ is

$$
\hat{u}_{n}(\xi)=\sum_{l} \sum_{j} c_{l}^{n}(j) h_{n} e^{-i h_{n} j \xi} \hat{\varphi}_{l}\left(h_{n} \xi\right), \quad \xi \in \mathbb{R} .
$$

It follows that for a nonnegative integer $\alpha$,

$$
\frac{(-i D)^{\alpha}}{\alpha !} \hat{u}_{n}(\xi)=h_{n}^{\alpha} \sum_{l=1}^{N} \sum_{\beta=0}^{\alpha} \frac{(-i D)^{\beta}}{\beta !} \hat{\varphi}_{l}\left(h_{n} \xi\right) v_{l, \alpha-\beta}^{n}(\xi),
$$

where

$$
v_{l, \gamma}^{n}(\xi):=h_{n} \sum_{j} c_{l}^{n}(j) e^{-i h_{n} j \xi} j \gamma / \gamma !
$$

Now condition (1) of Lemma 5 implies

$$
\lim _{n \rightarrow \infty} \sum_{l=1}^{N} \hat{\varphi}_{l}(0) v_{l, 0}^{n}(0)=1 .
$$

Moreover, condition (2) of Lemma 5 yields the following:

$$
\lim _{n \rightarrow \infty} \sum_{l=1}^{N} \sum_{\beta=0}^{\alpha} \frac{(-i D)^{\beta}}{\beta !} \hat{\varphi}_{l}(2 \pi j) v_{l, \alpha-\beta}^{n}(0)=0 \quad \text { for } j \in \mathbb{Z} \backslash\{0\} \text { and } \alpha<k .
$$

Comparing the above two formulas with formulas (4) and (5) in [3], we conclude from the proof given there that there exists a function $\psi$ which is a linear combination of $\varphi_{l},(l=1, \ldots, N)$, and their integer translates such that $\hat{\psi}(0)=1$ and $(3.1)$ holds.

Now we are in a position to state the main result of this paper.

Theorem 2. Let $S$ be a space of functions on $\mathbb{R}$ with the above properties (i), (ii), and (iii). Then the following are equivalent:

( $\left.1^{\circ}\right) S$ contains a compactly supported function $\psi$ such that $\hat{\psi}(0)=1$ and $D^{\alpha} \hat{\psi}(2 \pi j)=0$ for $0 \leq \alpha<k$ and $j \in \mathbb{Z} \backslash\{0\}$.

$\left(2^{\circ}\right) S$ contains a compactly supported function $\psi$ such that the mapping $I-L_{\psi}$ is degree-reducing on $\pi_{k-1}$.

$\left(3^{\circ}\right) S$ provides approximation of order $k$ in $L^{p}$. 
Proof. The proof for $\left(1^{\circ}\right) \Rightarrow\left(2^{\circ}\right) \Rightarrow\left(3^{\circ}\right)$ is well known (see, e.g., [5,6, and 3]). It remains to prove that $\left(3^{\circ}\right)$ implies $\left(1^{\circ}\right)$. For this purpose, we consider the centered $B$-spline of order $k+2$ :

$$
M_{k+2}(t):=\left[-\frac{k+2}{2},-\frac{k}{2}, \ldots, \frac{k+2}{2}\right](\cdot-t)_{+}^{k+1}, \quad t \in \mathbb{R} .
$$

We write $u$ for $M_{k+2}$. Let $q$ be the polynomial given in Theorem 1. This polynomial can be factored as

$$
q(t)=p(t)(1-t)^{r} \quad(r \geq 0)
$$

with $p(1) \neq 0$. Without loss of any generality, we assume that

$$
p(1)=1 \text {. }
$$

We claim that there exists a function $f \in C_{c}^{k}(\mathbb{R})$ such that

$$
\left.\nabla^{r} f\right|_{[-k-4, k+4]}=u \text {. }
$$

First, we observe that the function $v$ given by

$$
v:=u+u(\cdot-1)+u(\cdot-2)+\cdots
$$

vanishes on $(-\infty,-(k+2) / 2)$ and satisfies $\nabla v=u$. Repeating this process $r$ times, we can find $g \in C^{k}(\mathbb{R})$ such that $\nabla^{r} g=u$. However, this $g$ is not compactly supported. We may truncate $g$ by multiplying it by a function $\chi \in C_{c}^{k}(\mathbb{R})$ which takes value 1 on $[-r-k-5, r+k+5]$. Let $f:=g \chi$. Then $f \in C_{c}^{k}(\mathbb{R})$ and satisfies $(3.2)$.

For the proof of $\left(3^{\circ}\right) \Rightarrow\left(1^{\circ}\right)$, we shall prove that $\left(1^{\circ}\right)$ holds under the following assumption,

$$
\lim _{h \rightarrow 0} \operatorname{dist}\left(f, S^{h}\right) / h^{k-1}=0,
$$

which is weaker than $\left(3^{\circ}\right)$. Thus our proof covers the result of de Boor and DeVore in [1]. Moreover, it is clear from this proof that the order of approximation of $S$ is always an integer. So we suppose that there exists a positive function $\varepsilon$ of $h, h>0$, such that $\varepsilon(h)$ converges to 0 as $h$ goes to 0 , and that

$$
\operatorname{dist}\left(f, S^{h}\right)<\varepsilon(h) h^{k-1} .
$$

Hence there exists $u_{h} \in S^{h}$ such that

$$
\left\|f-u_{h}\right\| \leq \varepsilon(h) h^{k-1} .
$$

It follows from the above inequality that

$$
\left\|p\left(T_{h}\right) \nabla^{r} f-p\left(T_{h}\right) \nabla^{r} u_{h}\right\| \leq \text { const } \varepsilon(h) h^{k-1},
$$


with const independent of $h$. Here and afterwards, const always means a positive constant, which may be different in different contexts. Assume that $n=h^{-1}$ is an integer bigger than $\max \{\operatorname{deg} p, \mu R\}$. From (3.2) we deduce that

$$
\left.p\left(T_{h}\right) \nabla^{r} f\right|_{[-k-3, k+3]}=p\left(T_{h}\right) u .
$$

We observe that

$$
\nabla=I-T=I-T_{h}^{n}=\left(I-T_{h}\right)\left(I+T_{h}+\cdots+T_{h}^{n-1}\right) .
$$

Hence, by Theorem 1, we have

$$
p\left(T_{h}\right) \nabla^{r} u_{h}=\left(I+T_{h}+\cdots+T_{h}^{n-1}\right)^{r}\left(q\left(T_{h}\right) u_{h}\right) \in S_{\mathrm{loc}}^{h} .
$$

But $S_{\text {loc }}^{h}$ is spanned by $\varphi_{i}(\dot{\bar{h}}-j), i=1, \ldots, N, j \in \mathbb{Z}$; therefore we can find sequences $\left(c_{i}^{h}(j)\right)_{j \in \mathbb{Z}}$ so that

$$
p\left(T_{h}\right) \nabla^{r} u_{h}=\sum_{i=1}^{N} \sum_{j \in \mathbb{Z}} c_{i}^{h}(j) \varphi_{i}(\dot{\bar{h}}-j) .
$$

This in connection with (3.3) and (3.4) gives us the following estimate:

$$
\left\|p\left(T_{h}\right) u-\sum_{i, j} c_{i}^{h}(j) \varphi_{i}(\dot{\bar{h}}-j)\right\|_{[-k-3, k+3]} \leq \operatorname{const} \varepsilon(h) h^{k-1} .
$$

Since supp $u \subseteq[-k-1, k+1], p\left(T_{h}\right) u$ vanishes outside $[-k-2, k+2]$. Thus it follows from (3.5) that

$$
\left\|\sum_{i, j} c_{i}^{h}(j) \varphi_{i}(\dot{\bar{h}}-j)\right\|_{[-k-3,-k-2] \cup[k+2, k+3]} \leq \operatorname{const} \varepsilon(h) h^{k-1},
$$

or equivalently,

$$
\left\|\sum_{i, j} c_{i}^{h}(j) \varphi_{i}(\cdot-j)\right\|_{h^{-1}([-k-3,-k-2] \cup[k+2, k+3])} \leq \operatorname{const} \varepsilon(h) h^{k-1} .
$$

Now we set

$$
\begin{aligned}
& E_{1}:=\left\{(i, j): \operatorname{supp} \varphi_{i}(\cdot-j) \subseteq h^{-1}(-k-3, k+3)\right\}, \\
& E_{2}:=\left\{(i, j): \operatorname{supp} \varphi_{i}(\cdot-j) \cap\left(h^{-1}(-k-3, k+3)\right)=\varnothing\right\}, \\
& E_{3}:=\{(i, j): i=1, \ldots, N, j \in \mathbb{Z}\} \backslash\left(E_{1} \cup E_{2}\right) .
\end{aligned}
$$

Since $\varphi_{1}, \ldots, \varphi_{N}$ are supported on $[0, \mu R]$, we have, for $(i, j) \in E_{3}$, $\operatorname{supp} \varphi_{i}(\cdot-j) \cap\left(\left(\frac{-k-3}{h}, \frac{-k-3}{h}+\mu R\right) \cup\left(\frac{k+3}{h}-\mu R, \frac{k+3}{h}\right)\right) \neq \varnothing$. 
Noting that $\mu R \leq h^{-1}$, we invoke Lemma 4 and deduce from (3.6) that

$$
\left|c_{i}^{h}(j)\right| \leq \operatorname{const} \varepsilon(h) h^{k-1} \quad \text { for all }(i, j) \in E_{3} .
$$

Let

$$
v_{h}:=\sum_{(i, j) \in E_{1}} c_{i}^{h}(j) \varphi_{i}(\dot{\bar{h}}-j),
$$

and

$$
g:=p\left(T_{h}\right) u-v_{h} .
$$

Then $v_{h}$ vanishes outside $(-k-3, k+3)$, and so does $g$. Furthermore, when $(i, j) \in E_{2}$, the function $\varphi_{i}(\dot{\bar{h}}-j)$ vanishes on $(-k-3, k+3)$; hence for $x \in(-k-3, k+3)$,

$$
\sum_{i, j} c_{i}^{h}(j) \varphi_{i}\left(\frac{x}{h}-j\right)=\sum_{(i, j) \in E_{1}} c_{i}^{h}(j) \varphi_{i}\left(\frac{x}{h}-j\right)+\sum_{(i, j) \in E_{3}} c_{i}^{h}(j) \varphi_{i}\left(\frac{x}{h}-j\right) .
$$

By (3.7), we have

$$
\left\|\sum_{(i, j) \in E_{3}} c_{i}^{h}(j) \varphi_{i}\left(\frac{\dot{ }}{h}-j\right)\right\|_{(-k-3, k+3)} \leq \operatorname{const} \varepsilon(h) h^{k-1} .
$$

This together with (3.5) yields the following estimate:

$$
\|g\| \leq \text { const } \varepsilon(h) h^{k-1} \text {. }
$$

Consider the Fourier-Laplace transform of $g$ :

$$
\hat{g}(z):=\int_{\mathbb{R}} e^{-i z x} f(x) d x, \quad z \in \mathbb{C} .
$$

As we did in [3], we can invoke Cauchy's formula to get the estimate

$$
\left\|D^{\alpha} \hat{g}\right\|_{\infty}(\mathbb{R}) \leq \text { const } \varepsilon(h) h^{k-1}, \quad 0 \leq \alpha<k .
$$

The Fourier-Laplace transform of $p\left(T_{h}\right) u$ is

$$
\left(p\left(T_{h}\right) u\right)^{\wedge}(z)=p\left(e^{-i h z}\right) \hat{u}(z),
$$

with

$$
\hat{u}(z)=\left(\frac{\sin z / 2}{z / 2}\right)^{k+2} .
$$

Since $p(1)=1$, we have

$$
\left(p\left(T_{h}\right) u\right)^{-}(0)=1 .
$$

Moreover, it is easy to verify the following:

$$
\lim _{h \rightarrow 0} \frac{D^{\alpha}\left(p\left(T_{h}\right) u\right)^{\wedge}(\xi / h)}{h^{k-1}}=0 \text { for } \xi \in \mathbb{R} \backslash\{0\} \text { and } 0 \leq \alpha<k .
$$

Let $h_{n}=1 / n, n=1,2, \ldots$, and $v_{n}=v_{h_{n}}$. From (3.8)-(3.11) we conclude that the sequence $\left(v_{n}\right)$ satisfies the hypothesis of Lemma 5 . Therefore by this 
lemma we can find a compactly supported function $\psi \in S$ such that $\hat{\psi}(0)=1$ and $D^{\alpha} \hat{\psi}(2 \pi j)=0$ for $0 \leq \alpha<k$ and $j \in \mathbb{Z} \backslash\{0\}$. The proof is complete.

\section{ACKNOWLEDGMENT}

The author wishes to thank Professor S. Riemenschneider for his many valuable suggestions and comments on this paper.

\section{REFERENCES}

1. C. de Boor and R. DeVore, Partitions of unity and approximation, Proc. Amer. Math. Soc. 93 (1985), 705-709.

2. C. de Boor and K. Höllig, Bivariate box splines and smooth pp functions on a three direction mesh, J. Comput. Appl. Math. 9 (1983), 13-28.

3. C. de Boor and R.-Q. Jia, Controlled approximation and characterization of the local approximation order, Proc. Amer. Math. Soc. 95 (1985), 547-553.

4. R. Q. Jia, A counterexample to a result concerning controlled approximation, Proc. Amer. Math. Soc. 97 (1986), 647-654.

5. I. J. Schoenberg, Contributions to the problem of approximation of equidistant data by analytic functions, Parts A and B, Quart. Appl. Math. 4 (1946), 45-99, 112-141.

6. G. Strang and G. Fix, A Fourier analysis of the finite element variational method, Constructive Aspects of Functional Analysis (G. Geymonat, ed.), CIME, Ciclo, 1973, pp. 793-840. CHINA

Current address: Department of Mathematics, University of Oregon, Eugene, Oregon 97403 